\title{
ORBIT CONTROL AT THE SLS STORAGE RING
}

\author{
M. Böge, J. Chrin, P. Pollet, T. Schilcher, V. Schlott, PSI, Villigen, Switzerland
}

\begin{abstract}
Precise orbit control is one of the crucial ingredients for stable operation of the SLS storage ring. The orbits are taken by the digital BPM system which allows beam position measurements to the sub-micron level at sampling rates of up to $4 \mathrm{kHz}$ at 72 locations in the ring. Orbits are corrected with respect to a user defined reference by applying SVD techniques and a direct response matrix inversion. A slow global orbit feedback operating at correction rates of up to $1 \mathrm{~Hz}$ stabilizes the orbit to within $\sim 0.5 \mu \mathrm{m} \mathrm{rms}$ at the locations of the insertion devices. Energy drifts are automatically corrected using the RF frequency as an additional corrector, resulting in a long term energy stability of $\sigma(\mathrm{dP} / \mathrm{P}) \approx 10^{-5}$. The status of a digital BPM system based fast orbit feedback running at $4 \mathrm{kHz}$ sampling rate is presented.
\end{abstract}

\section{INTRODUCTION}

It is vital for a successful user operation to reproduce and stabilize a previously established reference orbit ("Golden Orbit") within 1/10th of the vertical beam size corresponding to $\approx 1 \mu \mathrm{m}$ at the location of the insertion devices (IDs). As a consequence, the digital BPM [1] and corrector hardware [2] have been designed in order to allow for the implementation of a Fast Orbit Feedback (FOFB) that is able to suppress residual orbit oscillations up to $\approx 100 \mathrm{~Hz}$ with attenuation factors of $>10$. The FOFB imposes tight constraints on the capabilities and reliability of the involved subsystems since the feedback loop runs at a sampling rate of $4 \mathrm{kHz}$ on the DSP level of the BPM hardware.

In order to gain experience with the various subsystems, a Slow Orbit Feedback (SOFB) with much relaxed requirements $(<3 \mathrm{~Hz}$ correction rate) has been implemented, communicating with the underlying hardware components through a "CDEV Server" which is connected to the EPICS based control system [3].

\section{GLOBAL ORBIT CORRECTION AND SOFB}

Global closed orbit correction in the SLS storage ring is based on the Singular Value Decomposition (SVD) technique which makes "Most Effective Corrector" and "MICADO" like long range correction schemes obsolete. For the horizontal orbit correction it is crucial to take into account path-length effects by correcting off-energy orbits with the RF frequency. The difference of the original orbit and the SVD fitted off-energy part, with a deviation of $\mathrm{dP} / \mathrm{P}$ corresponding to a frequency change $\mathrm{df}$, is submitted to the orbit correction.
Since the beginning of the commissioning, this scheme has been successfully applied to perform operator induced corrections. Due to the modularity of the beam dynamics software environment [4], thoroughly tested software components could be reused to implement a Slow Orbit Feedback (SOFB). In this case the operator is "replaced" by a client program "Feedback Client" which initiates an orbit correction at a given frequency (see Fig. 1).

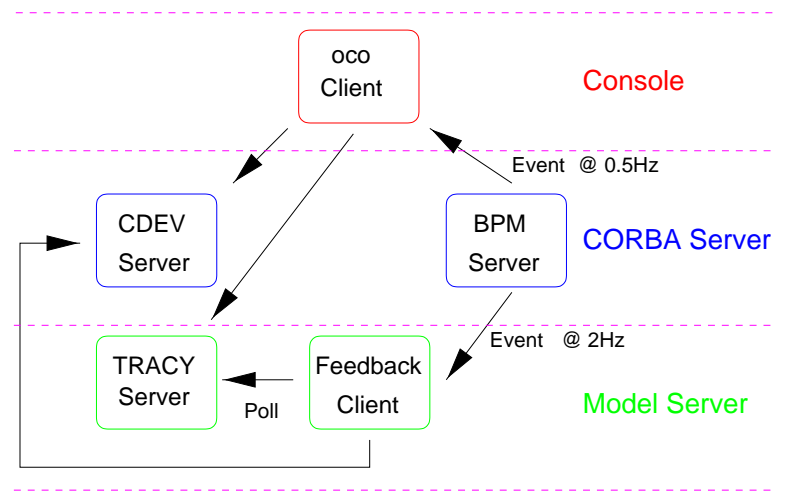

Figure 1: Schematic View of the SOFB: the "Feedback Client" on the "Model Server" level replaces the operator driven GUI "oco Client" on the "Console" level. The client gets BPM data from the "BPM Server", asks the "TRACY Server" for the corresponding model predicted corrector settings and applies the correction through the "CDEV Server"

\section{SOFB IMPLEMENTATION}

For the SOFB the digital BPM system is operated in an injection triggered batch processing mode with $32 \mathrm{kHz}$ bandwidth. By averaging over 64 orbit samples, corresponding to an interval of $2 \mathrm{~ms}$, it is possible to get "stroboscopic" position readings at a rate of $3 \mathrm{~Hz}$ with an error $<0.5 \mu \mathrm{m}$. The "BPM Server" in Fig. 1 monitors, collects and sends the BPM data to the "Feedback Client" with $2 \mathrm{~Hz}$. BPMs disabled on the EPICS level are automatically excluded from the SOFB loop. The same holds for correctors which have reached their maximum strength. The "TRACY Server" predicts a corrector pattern which restores the "Golden Orbit" defined by the "oco Client". Finally, the "CDEV Server" applies the proposed correction, which is toggling between the horizontal and vertical planes. Fine tuning of the feedback loop is done through a low pass filter, correcting on the average over several successive BPM data sets, and gain adjustment. By default the SOFB averages over 3 data sets and applies $75 \%$ of the proposed correction. Thus a full SOFB cycle takes $3 \mathrm{~s}$. It remains to be added that the SOFB runs only for one pre- 
viously set BPM gain. This ensures that transients visible at current induced BPM gain changes are not seen by the SOFB.

\section{SOFB RESULTS}

Fig. 2 documents the performance of the SOFB over 12 hours of user operation. After accumulating a current of $320 \mathrm{~mA}$, the top-up mode is entered [5, 6]. During 9 hours of top-up operation the SOFB stabilizes the orbit to rms values of $\approx 1 \mu \mathrm{m}$ with respect to the "Golden Orbit" in both planes. The RF frequency is corrected by df whenever $|\mathrm{df}|$ exceeds $5 \mathrm{~Hz}$ corresponding to $\mathrm{dP} / \mathrm{P} \approx 2 \cdot 10^{-5}$. In this particular case a frequency correction is performed every $\approx 45 \mathrm{~min}$. Please note that the horizontal $\mathrm{rms}$ value increases (see "saw tooth" in Fig. 2) while df is not applied. Fig. 3 shows the variation of the orbit position at

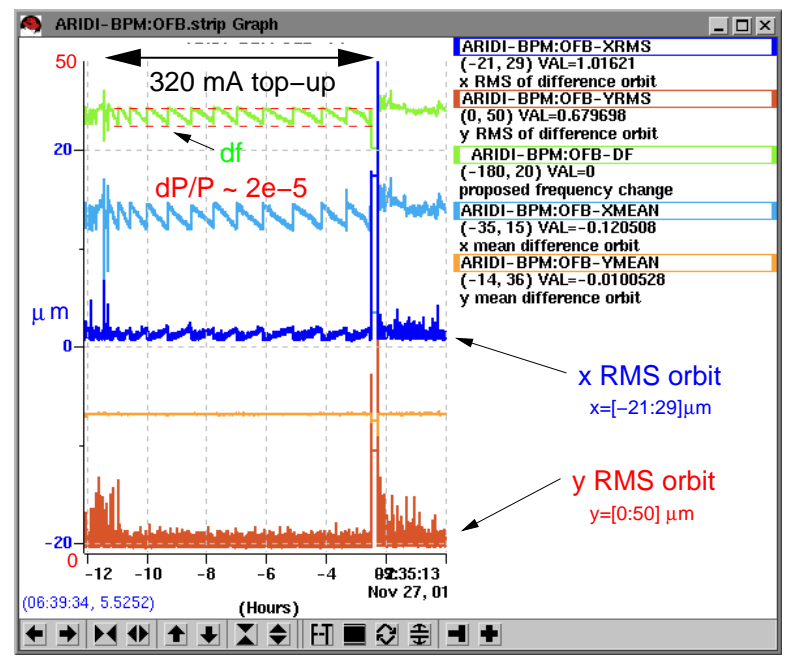

Figure 2: Screenshot depicting rms values of the horizontal ("x RMS") and vertical ("y RMS") deviations from the "Golden Orbit" of $\approx 1 \mu \mathrm{m}$ during a 9 hour "top-up" run at $320 \mathrm{~mA}$. The proposed frequency correction df is applied when passing a threshold of $5 \mathrm{~Hz}$ corresponding to an energy deviation $\mathrm{dP} / \mathrm{P} \approx 2 \cdot 10^{-5}$

the location of ID $\mathbf{U} 24$ over 13 hours of top-up operation. The values are extrapolated from the readings of the adjacent BPM upstream. The deviations from the "Golden Orbit" are well fitted by Gaussian distributions with 2nd moments of $\sigma_{x}=0.5 \mu \mathrm{m}$ and $\sigma_{y}=0.7 \mu \mathrm{m}$, respectively. The long term energy stability of the storage ring, characterized by the $\mathrm{dP} / \mathrm{P}$ change proposed by the SOFB, is shown in Fig. 4 which was recorded over 14 days of nearly continuous SOFB operation at a rate of $1 \mathrm{Sample} / \mathrm{min}$. During this period rms orbit deviations of $1 \mu \mathrm{m}$ could be maintained.

\section{FOFB IMPLEMENTATION}

In contrast to the $\mathrm{SOFB}$, which runs on a central PC, the global FOFB is decentralized and integrated in the twelve BPM stations of the SLS storage ring. The feedback will operate with an update rate of $4 \mathrm{kHz}$. Each of the twelve
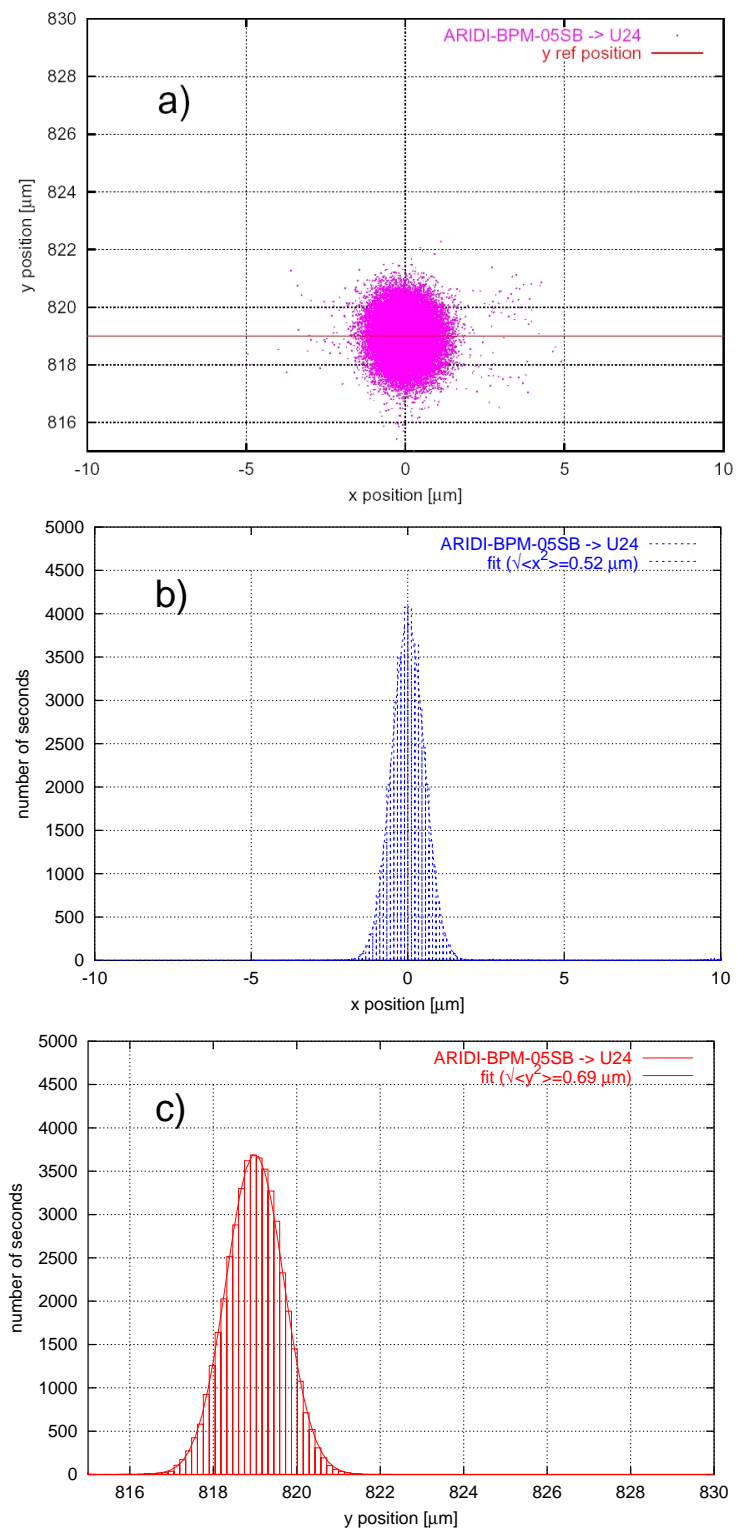

Figure 3: Scatter-plot a) of the projected orbit position at the location of ID U24 taken at a sampling rate of $1 \mathrm{~Hz}$ over a 13 hour top-up run and corresponding histograms for the horizontal b) and vertical c) beam motion. The 2nd moments of the Gaussian distributions are fitted to be $\sigma_{x}=0.5 \mu \mathrm{m}$ and $\sigma_{y}=0.7 \mu \mathrm{m}$, respectively

stations handles six BPM inputs and six corrector magnet outputs. The design of the FOFB at an early stage in the SLS project made it possible to combine the feedback with the digital BPM system without much additional expenditure. The hardware implementation of one of the twelve BPM stations is shown in Fig. 5. The six BPM position readings $(x, y)$ of the sector are already available in a digital format on a first digital signal processor (DSP 1). They are transfered over a multiprocessor bus to a second processor (DSP 2) which is devoted to the orbit feedback. Since the coefficients of the inverted response matrix only have significant values around the diagonal it is sufficient to 


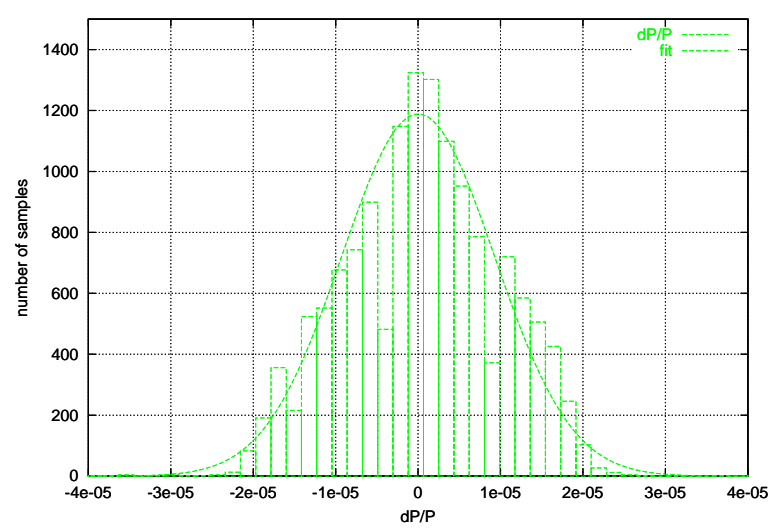

Figure 4: Energy stability (dP/P change by SOFB) over 14 days (1 Sample/min) of SOFB operation. The 2 nd moment of the Gaussian distribution is fitted to be $\sigma(\mathrm{dP} / \mathrm{P}) \approx 10^{-5}$

calculate the new corrector magnet settings with the adjacent BPM readings [7]. The required beam positions from the neighboring sectors are exchanged over a dedicated fibre optic point-to-point network. It uses the SHARC link ports to which a fibre optic module is attached. Separate channels for transmit and receive have been chosen between each sector thus avoiding any network collisions. The whole processing chain is driven by the data acquisition of the digital BPM system which in turn receives hardware triggers from the timing system. An interrupt is generated on DSP 1 as soon as data arrives from the digital receivers. After transferring the position readings to DSP 2 an interrupt is triggered to start the data exchange over the fibre links with four parallel DMA processors. Data packets of 56 Bytes are transmitted containing additional start and stop bytes. A timer monitors whether the adjacent sectors have responded within a predefined time window. Measurements in the laboratory between two VME crates have shown that the full data exchange requires in the order of $8 \mu \mathrm{s}$. Once the full set of BPM data of three sectors is available the predefined reference orbit is subtracted resulting in the final beam displacement. The sub-matrix multiplication yields the incremental corrector magnet changes which are then written over the VME bus to the power supply controller interface board. The VME bus master capability of the DSP board avoids any necessary intervention of the control system IOC. From the interface boards the new corrector settings are transfered star like to six digital power supply controllers over fibre optic links operating at $5 \mathrm{Mbits} / \mathrm{s}$.

\section{FOFB STATUS}

Up to now, the digital BPM/FOFB system and the corrector magnet system have been located in two different VME crates. This approach allowed an independent commissioning of two newly developed systems. Since the operation of the SOFB has already fulfilled the requirements of the experiments for the time being, low priority has been given to the implementation of the FOFB. On the other hand, operation of the SLS during the last couple of months has shown the importance of an "intelligent" BPM system which is capable of self diagnosing hardware faults. This is an important requirement for a reliable operation of the feedback system. Therefore emphasis has been put on sophisticated low level software in order to detect BPMs with spurious bad readings. When the feedback loop is closed the residual orbit fluctuations are typically in the order of $\mu \mathrm{m}$. If both rms values of $x$ and $y$ taken over the last 64 orbit samples exceed a predefined threshold an operator alarm is raised and the DSP software disables the possibly faulty BPM. Since the orbit feedback monitors the on-line status of all BPMs it automatically recalculates the inverse response matrix of the remaining set of BPMs and correctors and continues operation automatically. The next step in the implementation of the FOFB system is to shift the functionality of the SOFB from the central beam dynamics PC down to the DSP level and operate the feedback with initially $3 \mathrm{~Hz}$ before switching to the full sample rate of 4 kHz.

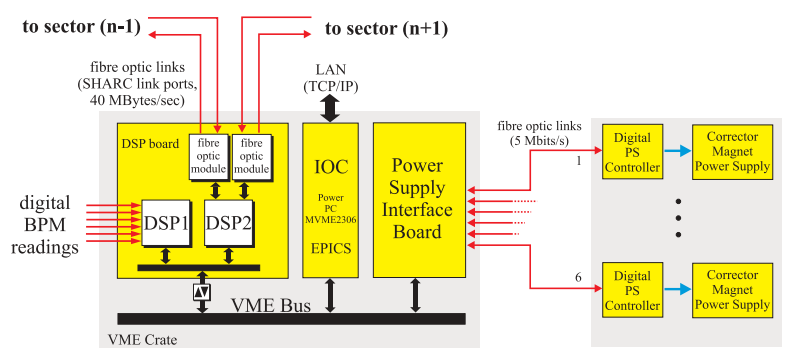

Figure 5: Hardware layout of the FOFB in one of the twelve sectors

\section{CONCLUSION}

Short and long term orbit stabilities of $<1 \mu \mathrm{m}$ at the location of the IDs and an energy stability of $\sigma(\mathrm{dP} / \mathrm{P}) \approx 10^{-5}$ have been achieved. The successful commissioning of the SOFB is an important step towards the implementation of the FOFB.

\section{REFERENCES}

[1] V. Schlott et al., "Commissioning of the SLS Digital BPM System", PAC'01, Chicago 2001.

[2] L. Tanner, F. Jenni, "Digitally Controlled SLS Magnet Power Supplies", PAC'01, Chicago 2001.

[3] S. Hunt et al., "Control and Data Acquisition System of the SLS”, ICALEPCS'99, Trieste 1999.

[4] M. Böge et al., "Commissioning of the SLS using CORBA Based Beam Dynamics Applications", PAC'01, Chicago 2001.

[5] A. Streun et al., "Commissioning of the Swiss Light Source", PAC'01, Chicago 2001.

[6] M. Muñoz et al., "Top-up Operation at the Swiss Light Source", these proceedings

[7] M. Böge et al., "Fast Closed Orbit Control in the SLS Storage Ring”, PAC'99, New York 1999. 